\title{
Security and Privacy in New Computing Environments (SPNCE 2016)
}

\author{
Jin $\mathrm{Li}^{1} \cdot$ Xiaofeng $\mathrm{LiaO}^{2}$
}

Accepted: 27 September 2021 / Published online: 8 January 2022

(c) The Author(s), under exclusive licence to Springer Science+Business Media, LLC, part of Springer Nature 2021

\section{Editorial:}

The existing computing models and computing environments have changed immensely due to the rapid advancements in mobile computing, big data, and cyberspace-based supporting technologies such as cloud computing, Internet-of-Things and other large-scale computing environments. For example, cloud computing is an emerging computing paradigm in which IT resources and capacities are provided as services over the Internet. It builds on the foundations of distributed computing, grid computing, virtualization, service orientation, etc. Cloud computing offers numerous benefits from both the technology and functionality perspectives such as increased availability, flexibility, and functionality. Traditional security techniques are faced many challenges in these new computing environments. Thus, efforts are needed to explore the security and privacy issues of the above-mentioned new environments within the cyberspace.

In this special issue, we selected 8 papers with rigorous review process. Our selection is based on the relevance to the special issue topics, paper quality, methods and research contributions. This special issue has selected the following papers:

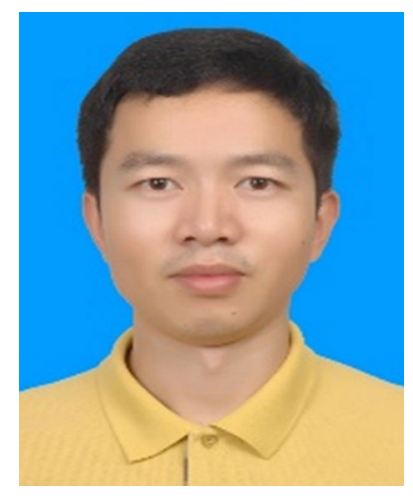

Jin Li

lijin@gzhu.edu.cn

1 Guangzhou University, Guangzhou, China

2 College of Computer Science, Chongqing University, Chongqing, China
One of the most important issues is Secure Cloud Storage in New Computing Environments. In the paper entitled "Verifiable public-key encryption with keyword search secure against continual memory attacks", the authors constructed a verifiable PEKS scheme which can efficiently verify the completeness of the result from the tester and thus the correctness. In the paper entitled "A Novel Visual Medical Image Encryption for Secure Transmission of Authenticated Watermarked Medical Images", the authors proposed a visual medical image encryption technique to protect the existence of the watermarked medical image. And to ensure the source authentication, a biometric based authentication is also proposed. In the paper entitled "A fine-grained attribute based data retrieval with proxy re-encryption scheme for data outsourcing systems", the authors presented an attribute based date retrieval with proxy re-encryption (ABDR-PRE) to provide both fine-grained access control and retrieval over the ciphertexts.

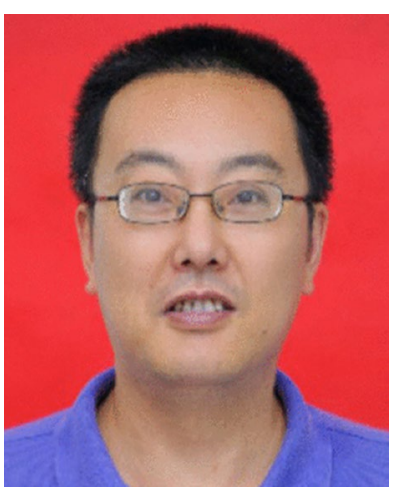

Security in IoT is another important security issue in New Computing Environments. In paper "Fine-grained Trust-based Routing Algorithm for Wireless Sensor Networks", the authors proposed a high-reliability trust evaluation model for secure routing based on combination inside states of a node with outside interaction behaviors between nodes. In paper "New engineering method for the Risk assessment: Case Study Signal Jamming of the m-health 
Networks", the authors proposed a novel framework to facilitate the risk analysis and adopted a methodology based on four steps: the assessment, the classification, the archiving, and the exchanging of risk parameters.

Access Control and Authentication is also an important problem in New Computing Environments. The paper "Fuzzy Logic with Expert judgment to Implement an Adaptive Riskbased Access Control Model for IoT", the authors proposed an adaptive risk-based access control model for the IoT. This model uses real-time contextual information associated with the requesting user to calculate the security risk regarding each access request. In paper "A survey of Android mobile phone authentication schemes", the authors presented an analysis of some of the authentication schemes that are used in mobile devices and some of the threats and technical issues faced.

There are also some other studies in New Computing Environments such as Remote Data Deduplication. In the paper entitled "Privacy in Cross-User Data Deduplication", the authors introduced a privacy requirement for data deduplication techniques called data-unlinkability to resolve this problem. Moreover, they propose a novel approach for data deduplication using Bloom filter to provide data-unlinkability for clients' files.

Acknowledgements The guest editors are thankful to our reviewers for their effort in reviewing the manuscripts.

Publisher's Note Springer Nature remains neutral with regard to jurisdictional claims in published maps and institutional affiliations.
Jin Li (Fellow, IEEE) is currently a Professor in Guangzhou University, Guangzhou. He served as a Senior Research Associate with the Korea Advanced Institute of Technology, Daejeon, South Korea, and the Illinois Institute of Technology, Chicago, IL, USA, from 2008 to 2010 , respectively. He has published more than 100 papers in international conferences and journals, including IEEE INFOCOM, the IEEE Transactions on Information Forensics and Security, the IEEE Transactions on Parallel and Distributed Systems, the IEEE Transactions on Computers, and the European Symposium on Research in Computer Security. His work has been cited more than 11,000 times on Google Scholar and his $\mathrm{H}$-index is 40 . His research interests include artificial intelligence, cloud computing, and cryptographic protocols.

Xiaofeng Liao (Fellow, IEEE) received the B.S. and M.S. degrees in mathematics from Sichuan University, Chengdu, China, in 1986 and 1992, respectively, and the Ph.D. degree in circuits and systems from the University of Electronic Science and Technology of China, Chengdu, in 1997. From 1999 to 2012, he was a Professor with Chongqing University, Chongqing, China. From 2012 to 2018, he was a Professor and the Dean of the College of Electronic and Information Engineering, Southwest University, Chongqing. He is currently a Professor and the Dean of the College of Computer Science, Chongqing University. He is also a Yangtze River Scholar of the Ministry of Education of China, Beijing, China. From 1997 to 1998, he was a Research Associate with the Chinese University of Hong Kong, Hong Kong. From 1999 to 2000, he was a Research Associate with the City University of Hong Kong, Hong Kong. From March to June 2001 and March to June 2002, he was a Senior Research Associate with the City University of Hong Kong. From 2006 to 2007, he was a Research Fellow with the City University of Hong Kong. He holds five patents, and published four books and over 300 international journal and conference papers. His current research interests include optimization and control, machine learning, privacy protection, neural networks, and bifurcation and chaos. Dr. Liao currently an Associate Editor for the IEEE Transactions on Cybernetics and IEEE Transactions on Neural Networks and Learning Systems. 